\title{
Influence of reagent formulation on mRNA quantification by RT-PCR using imported external standard curves
}

\author{
Mireia Farriol ${ }^{\bowtie}$ and Xavi Orta \\ Centro de Investigaciones Biológicas y Biología Molecular, (CIBBIM), Hospital General Vall d'Hebron,

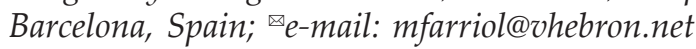

Received: 10 December, 2004; revised: 30 March, 2005; accepted: 12 April, 2005

available on-line: 11 July, 2005

\begin{abstract}
Use of an imported external standard curve is common in real-time quantitative RT-PCR. Two practical strategies for long-term experiments include importing a grand mean standard curve to all accumulated runs or using daily imported standard curves, fixing the slope at the beginning of the experiment and calibrating successive runs with curves generated from this imported slope, adding a single standard that registers the variation in the y-intercept. This study determines the influence that a change in reagent lots has on these two calibration approaches when determining mRNA copy numbers of the ornithine decarboxylase and porphobilinogen deaminase genes. Two sets of determinations were run with the use of lot A and lot B. A marked decrease in the crossing points $(\mathrm{Cp})$ in the standards for both genes at all concentration levels was observed with the change in lots. A grand mean standard curve was generated for each gene and each set and comparisons between the sets were performed. Statistically significant differences were found with respect to the $y$-intercept but not the slope, suggesting that the change of reagent lot affected the detection sensitivity but not the efficiency of the reaction. The excellent correlation coefficients obtained for these curves for each gene were not achieved when overall data from both sets were combined to generate an overall grand mean standard curve. We conclude that when faced with a change of RT-PCR reagent lot that will affect the detection sensitivity of the method, samples should be calculated with either the daily imported standard curves or with the respective grand mean standard curve for each lot.
\end{abstract}

Keywords: mRNA quantification, RT-PCR, ornithine decarboxylase

Measurement of mRNA by real-time RTPCR is now a widely used method for investigating changes in gene expression (Johnsson et al., 2000; Brabender et al., 2001; Kuhne \& Oschmann, 2002; Ovstebo et al., 2003; Shantz, 2004). Protocols using real-time technology for absolute quantification and many of those for relative quantification are based on the use of standard curves to calculate the mRNA copy number in the samples. This method has some advantages over other options that do not incorporate a standard curve (Pffaf, 2001; Liu \& Saint, 2002), such as the easy of comparing results between experiments or laboratories. The main drawback is that a pure standard is required. Some standards are not available on the market and production of a pure standard in the laboratory is highly time-consuming. In addition, analysis of several dilutions of the standard and a housekeeping gene are required to obtain the respective calibra- tion curves, hence a larger number of samples has to be processed in each run.

To overcome these difficulties, some useful tools have been included in the protocols using imported standard curves. One such approach introduces a single calibration curve in each run for both the target gene and the housekeeping gene, but this option is only feasible when the amplification efficiencies of the two genes are very similar. Another practical strategy to simplify the calibration process consists in importing a single curve to successive runs, maintaining a constant slope and adding a single concentration of standard in each run in order to register the change in the y-intercept; this is the daily imported external curve. This mathematical system is now incorporated in the 3.5 version of the LightCycler software. Acceptance of this approach comes from studies of reproducibility showing that in different runs with an identical standard curve,

Abbreviations: $\mathrm{Cp}$, crossing point; $\mathrm{CV}$, coefficient of variation; h-PBGD, porphobilinogen deaminase; ODC, ornithine decarboxylase. 
the slope of the curve, which dictates the overall efficiency of the reaction $\left(\mathrm{E}=10^{-1 / \text { slope }}\right)$, presents a $\mathrm{CV}$ of $2-3 \%$, but this variation can be higher for the $y$ intercept, which can reach 10\% (Rasmunsen, 2001). Another calculation strategy is based on the use of a grand mean calibration curve, which is derived from accumulated data (one year) after confirming the reproducibility of the results (Ovstebo et al., 2003).

When using any of the calculation strategies mentioned, special precautions should be taken if the experimental conditions change. One typical change is that produced when a different reagent lot is used during the analysis of a large series. It has been demonstrated that this type of change can randomly affect the results by modifying kinetic parameters such as the detection sensitivity (Burgos et al., 2002).

The aim of this study was to investigate the influence of a change in reagent lots on two quantification strategies used with a method of calibration based on imported standard curves for the ODC and h-PBGD genes.

\section{MATERIAL AND METHOD}

Real-time quantitative RT-PCR was performed with Light-Cycler technology (Roche Molecular Biochemicals). The housekeeping gene porphobilinogen deaminase (h-PBGD) was used as an internal reference. The ornithine decarboxylase (ODC) RNA standard was made in our laboratory, as described (Orta et al., 2002), and the h-PBGD RNA standard was obtained commercially (Roche, Cat. No. 3146073).

The reaction mixture for ODC analysis contained $10 \times$ Light-Cycler RT-PCR mix hybridization probes plus $\mathrm{MgCl}_{2}$ to a final concentration of $6 \mathrm{mM}$, $0.5 \mu \mathrm{M}$ of each primer and $0.2 \mu \mathrm{M}$ of each probe. For h-PBGD analysis, we employed a commercially available ready-to-use reaction mixture containing primers and probes (final volume, $15 \mu \mathrm{L}$ ). The ODC primers used were as follows:

forward 5'-TgATTggATgCTCTTTgAAAACAT-3' and reverse 5'-ACACATTAATACTAgCCgAAgCAC-3'.

The ODC probes at $0.2 \mu \mathrm{M}$ final concentration included: fluoresceine $5^{\prime}$-TggAATTgCTgCATgAgTTgCCAC-3' and Red640 5'-TgCCCTgACATCACATAgTAgATCgTCggC-3'.

Reverse transcription was performed at $55^{\circ} \mathrm{C}$ for $10 \mathrm{~min}$ followed by denaturation at $95^{\circ} \mathrm{C}$ for $2 \mathrm{~min}$, and PCR amplification for 45 cycles (5 s at $95^{\circ} \mathrm{C}, 15 \mathrm{~s}$ at $57^{\circ} \mathrm{C}$ and $13 \mathrm{~s}$ at $72^{\circ} \mathrm{C}$ ).

Patients with colorectal carcinoma were the subjects of this study and the protocol was approved by the hospital ethics committee. Samples $(n=62)$ of both tumor tissue and adjacent non-tumor tissue weighing $30 \mathrm{mg}$ were obtained from the resected surgical specimen of each patient in the operating room. The tissues were immediately immersed in liquid nitrogen and stored at $-80^{\circ} \mathrm{C}$. The samples were analyzed in 18 different runs over a period of three months. At the beginning of the second month the reagent lot was changed and two sets of experiments were created, A and B, associated with the use of the two reagent lots. At the beginning of the experiment, a calibration curve was generated for each gene by measuring the standards in triplicate at different concentration levels: $1.2 \times 10^{7}, 1.2 \times 10^{6}$, $1.2 \times 10^{5}$ copies/ $\mu \mathrm{L}$ for the ODC calibrator and $10^{6}$, $10^{5}, 10^{4}$, for the h-PBGD calibrator. In the successive runs only one standard was measured for each gene, importing the slope of the initial curve for the calculation of mRNA concentration and updating the curve with the new y-intercept registered in each run. The LightCycler software, version 3.5, from Roche was used in the calculation and the curves for the two lot periods were compared.

Student's t-test for paired data, Wilcoxon's test and Spearman's correlation coefficient were used for statistical comparisons between the results. Statistical significance was set at a $P$-value of $<0.05$.

\section{RESULTS AND DISCUSSION}

Analysis of the crossing points (Cp) showed a systematic decrease in $\mathrm{Cp}$ values of around $8.5 \%$ at all concentrations in both genes with the change in reagent lot (Table 1). Statistical analysis applied by concentration levels confirmed the statistically significant differences in $\mathrm{Cp}$ values between periods A and B. Subsequently, for each of the periods, we calculated the respective coefficients of variation of the y-intercept of the daily imported external standard curves (Table 2). The values obtained were less than $1 \%$ and similar to those reported by other authors (e.g. Kühne, 2002). In contrast, with this good intraperiod reproducibility of the daily imported external standard curves, we found that the ranges of values of the $y$-intercept for the two periods did not overlap; those of period A were clearly higher than those of period B (Table 2).

To analyze the cause of this substantial shift of the $y$-intercept associated with the change of lot, we looked at the equation of the calibration curve:

$\mathrm{Cp}=-\left(1 / \log \mathrm{E} \cdot \log \mathrm{T}_{0}+(\log \mathrm{K} / \log \mathrm{E})\right.$

In this formula, the y-intercept is the result of dividing the logarithm of the "amount of product" of the PCR at the point of detection $(\mathrm{K})$ by the logarithm of the efficiency (E), implying that the $y$-intercept can be modified with or without a variation in the efficiency. Thus from a practical viewpoint it would be very useful to compare the grand mean cal- 
Table 1. Variation in Cp values of ODC and h-PBGD standards with two reagent lots.

Values expressed as mean \pm S.D. $\left({ }^{*}: P<0.05\right)$.

\begin{tabular}{lllll}
\hline & \multicolumn{3}{l}{ ODC } & h-PBGD \\
\cline { 2 - 5 } Log copy No. & Lot A & Lot B & Lot A & Lot B \\
\hline 7 & $22.16 \pm 0.18$ & $19.00 \pm 0.28^{*}$ & - & - \\
6 & $25.75 \pm 0.21$ & $23.00 \pm 0.41^{*}$ & $22.41 \pm 0.47$ & $20.34 \pm 0.24^{*}$ \\
5 & $29.33 \pm 0.24$ & $26.85 \pm 0.29^{*}$ & $25.65 \pm 0.39$ & $23.64 \pm 0.45^{*}$ \\
4 & - & - & $29.35 \pm 0.25$ & $27.11 \pm 0.22^{*}$ \\
\hline
\end{tabular}

ibration curve of both periods. This is important because it is the basis for proper use of the calibration data for measuring mRNA.

When we generated the grand mean calibration curve for each gene with all the points stored in the runs for period A, we obtained excellent correlation coefficients of 0.998 (ODC) and 0.992 (h-PBGD). The same occurred with the grand mean calibration curves for period B, with correlation coefficients of 0.994 (ODC) and 0.995 (h-PBGD). Nevertheless, and as was expected from the variation of $C p$ values, when we attempted to generate a global grand mean calibration curve (period A + period B), we obtained correlation coefficients of 0.90 (ODC) and 0.93 (hPBGD), much lower than 0.99, the value required for a reliable quantitative analysis.

For both ODC and h-PBGD mRNA calculations, a statistical comparison of the slopes for the two periods (using a program for analysis of covariance at http://home.ubalt.edu/ntsbarsh) demonstrated statistically significant differences in the y-intercept but not in the slope (Fig. 1). That is, the change in reagent lot affected the detection sensitivity (amount of product accumulated at the point of detection), but not the efficiency of the reaction. In fact, between one period and the other, the efficiencies differed by only 0.018 for ODC and 0.035 for h-PBGD, which is lower than 0.05 , the limit considered acceptable for use of a common calibration slope.

Thus, starting from an analysis of the stored data and knowing the effect of the change of lot on the calibration curve, the choice of a suitable strategy for calibration can be made. If a change is produced in the detection sensitivity without affecting the efficiency (slope), use of the daily imported external standard curves for the entire period would be appropriate. The alternative is to quantify with two grand mean curves, one for each lot.

To confirm the validity of these strategies we calculated the samples from the 62 patients included in the study using the daily imported mean and the grand mean from each period. Comparison of the number of copies of ODC mRNA and h-PBGD mRNA showed no significant differ-

Table 2. Reproducibility of ODC and h-PBGD daily imported standard curves

\begin{tabular}{|c|c|c|c|c|c|}
\hline & Run & $\mathrm{y}$-intercept ODC & Slope ODC & $y$-intercept PBGD & Slope PBGD \\
\hline \multirow[t]{7}{*}{ LOT A } & 1 & 46.32 & -3.405 & 42.70 & -3.34 \\
\hline & 2 & 46.08 & -3.405 & 41.82 & -3.34 \\
\hline & 3 & 46.00 & -3.405 & 42.44 & -3.34 \\
\hline & 4 & 45.52 & -3.405 & 42.09 & -3.34 \\
\hline & 5 & 46.54 & -3.405 & 42.88 & -3.34 \\
\hline & 6 & 46.39 & -3.405 & 42.35 & -3.34 \\
\hline & 7 & 46.81 & -3.405 & 41.69 & -3.34 \\
\hline $\mathrm{CV}(\%)$ & & 0.84 & & 0.96 & \\
\hline \multirow[t]{13}{*}{ LOT B } & 8 & 43.56 & -3.405 & 40.34 & -3.34 \\
\hline & 9 & 44.23 & -3.405 & 39.86 & -3.34 \\
\hline & 10 & 43.83 & -3.405 & 40.49 & -3.34 \\
\hline & 11 & 43.93 & -3.405 & 40.18 & -3.34 \\
\hline & 12 & 43.68 & -3.405 & 40.15 & -3.34 \\
\hline & 13 & 44.36 & -3.405 & 40.36 & -3.34 \\
\hline & 14 & 44.74 & -3.405 & 40.12 & -3.34 \\
\hline & 15 & 43.85 & -3.405 & 40.92 & -3.34 \\
\hline & 16 & 44.44 & -3.405 & 40.77 & -3.34 \\
\hline & 17 & 44.10 & -3.405 & 40.66 & -3.34 \\
\hline & 18 & 44.44 & -3.405 & 40.42 & -3.34 \\
\hline & 19 & 43.89 & -3.405 & 40.26 & -3.34 \\
\hline & 20 & 44.16 & -3.405 & 40.60 & -3.34 \\
\hline $\mathrm{CV}(\%)$ & & 0.75 & & 0.70 & \\
\hline
\end{tabular}




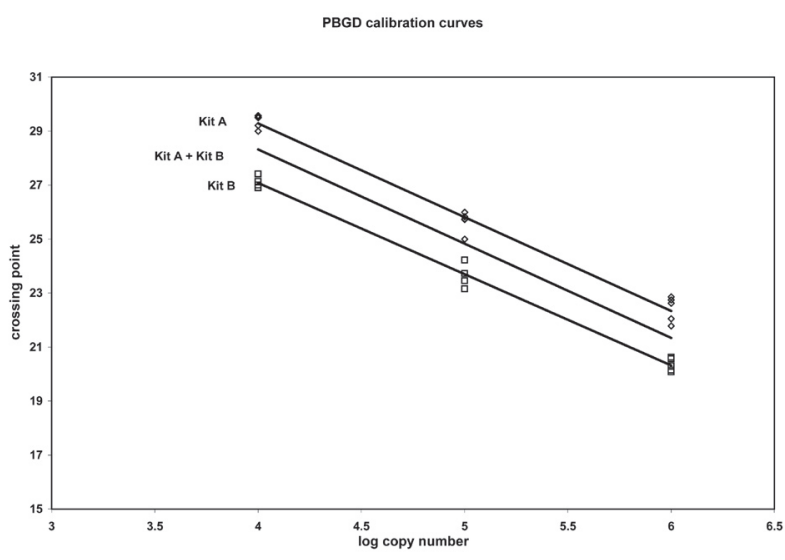

Figure 1. Differences in y-intercept.

ences (mean \pm S.E.); ODC: $1068089 \pm 193380$ vs. $1052553 \pm 184584$ and h-PBGD: $57317 \pm 7998$ vs. $59321 \pm 7943$. The correlation coefficients found were statistically significant for ODC $(r=0.980)$, h-PBGD ( $\mathrm{r}=0.979)$, and the ODC/h-PBGD ratio (0.996). Therefore, we believe that it is not suitable to calibrate with a global grand mean curve covering both periods. In contrast, the other option provided calibration data that could be used in a convenient manner for reliable mRNA quantitation.

\section{REFERENCES}

Brabender J, Lord RV, Daneneberg KD et al. (2001) Upregulation of ornithine decarboxylase mRNA expression in Barrett's esophagus and Barrett's associated adenocarcinoma. J Gastrointestinal Surg 5: 174-178.

Burgos JS, Ramirez C, Tenorio R, Sastre I, Bullido MJ (2002) Influence of reagents formulation on real-time PCR parameters. Mol Cell Probes 16: 257-260.

Johnson MR, Wang K, Smith JB, Heslin MJ, Diasio RB (2000) Quantitation of dihydropyrimidine dehydrogenase expression by real-time reverse transcription polymerase chain reaction. Anal Biochem 278: 175-184.

Kühne BS, Oschmann P (2002) Quantitative real-time RTPCR using hybridization probes and imported standard curves for cytokine gene expression analysis. Biotechniques 33: 1080-1084.

Liu W, Saint DA (2002) A new quantitative method of real time reverse transcription polymerase chain reaction assay based on simulation of polymerase chain reaction kinetics. Anal Biochem 302: 52-59.

Orta X, Balsells J, Olsina J, Venereo Y, Segovia-Silvestre T, Farriol M (2002) Quantitative analysis of ornithine decarboxylase mRNA by reverse transcription-PCR with the Light-Cycler system. Clin Chem 48: 1779-1781.

Ovstebo R, Foss KB, Lande K, Kierulf P (2003) PCR-based calibration curves for studies of quantitative gene expression in human monocytes development and evaluation. Clin Chem 49: 425-432.

Pfaffl MW (2001) A new mathematical model for relative quantification in real-time RT-PCR. Nucleic Acids Res 29: 2002-2027.

Rasmussen R (2001) Quantification on the LightCycler. In Rapid Cycle Real-Time PCR, Methods and Applications. Meuer S, Wittwer C, Nakagawara K, eds, pp 21-24. Springer Press, Heidelberg.

Rasmussen R (2004) In: http//www.idahotech.com/lightcycler_u/lectures.

Shantz LM (2004) Transcriptional and translational control of ornithine decarboxylase during Ras transformation. Biochem J 377 (Pt 1): 257-264. 